\title{
Familial Xp11.22 microdeletion including SHROOM4 and CLCN5 is associated with intellectual disability, short stature, microcephaly and Dent disease: a case report
}

\author{
Magdalena Danyel ${ }^{1}$, Eun Kyung Suk ${ }^{2}$, Vera Raile ${ }^{3}$, Jutta Gellermann ${ }^{4}$, Alexej Knaus ${ }^{5}$ and Denise Horn ${ }^{1 *}$ (D)
}

\begin{abstract}
Background: Two interstitial microdeletions Xp11.22 including the CLCN5 and SHROOM4 genes were recently reported in a male individual affected with Dent disease, short stature, psychomotor delay and minor facial anomalies. Dent disease, characterized by a specific renal phenotype, is caused by truncating mutations of CLCN5 in the majority of affected cases.

Case presentation: Here, we present clinical and molecular findings in a male patient with clinical signs of Dent disease, developmental delay, short stature, microcephaly, and facial dysmorphism. Using molecular karyotyping we identified a hemizygous interstitial microdeletion Xp11.23p.11.22 of about $700 \mathrm{~kb}$, which was inherited from his asymptomatic mother. Among the six deleted genes is CLCN5, which explains the renal phenotype in our patient. SHROOM4, which is partially deleted in this patient, is involved in neuronal development and was shown to be associated with X-linked intellectual disability. This is a candidate gene, the loss of which is thought to be associated with his further clinical manifestations.

To rule out mutations in other genes related to intellectual disability, whole exome sequencing was performed. No other pathogenic variants that could explain the phenotypic features, were found.
\end{abstract}

Conclusion: We compared the clinical findings of the patient presented here with the reported case with an Xp11.22 microdeletion including CLCN5 and SHROOM4 and re-defined the phenotypic spectrum associated with this microdeletion.

Keywords: Dent disease, CLCN5, SHROOM4

\section{Background}

Dent disease is a well-known X-linked renal phenotype which is characterized by progressive proximal renal tubulopathy with low molecular weight proteinuria, nephrocalcinosis and hypercalciuria [1]. The condition varies in degrees of severity and is present in clinically related forms such as X-linked nephrolithiasis or hypercalciuric nephrolithiasis. Female carriers are usually asymptomatic, but show mild proteinuria and hypercalciuria occurs in about

\footnotetext{
* Correspondence: denise.horn@charite.de

${ }^{1}$ Institute of Medical Genetics and Human Genetics, Charité -

Universitätsmedizin Berlin, corporate member of Freie Universität Berlin

Humboldt-Universität zu Berlin, and Berlin Institute of Health, Berlin, Germany Full list of author information is available at the end of the article
}

50\%. CLCN5 mutations which are scattered throughout the coding region were detected in these patients [2]. The majority of these mutations are predicted to result in a truncated protein. In about $15 \%$ of patients, OCRL1 mutations are responsible for Dent disease [3].

SHROOM4, also known as KIAA1202 was shown to be associated with X-linked mental retardation (XLMR) in several unrelated individuals [4]. Two unrelated females with mild/moderate intellectual disabilities were found to have balanced X;autosome translocations with Xp11.2 breakpoints which disrupt SHROOM4. Affected individuals of another family with a missense variant in this gene presented with severe intellectual disability, delayed

(c) The Author(s). 2019 Open Access This article is distributed under the terms of the Creative Commons Attribution 4.0 International License (http://creativecommons.org/licenses/by/4.0/), which permits unrestricted use, distribution, and reproduction in any medium, provided you give appropriate credit to the original author(s) and the source, provide a link to the Creative Commons license, and indicate if changes were made. The Creative Commons Public Domain Dedication waiver (http://creativecommons.org/publicdomain/zero/1.0/) applies to the data made available in this article, unless otherwise stated. 
or no speech development, seizures, and hyperactivity [4]. Further molecular analyses showed expression of the human SHROOM4 in adult and fetal brain structures, and indicated its role in the function of specific neuronal population of cells by encoding a protein involved in cytoskeletal architecture [5]. Using array-based comparative genomic hybridization (array $\mathrm{CGH}$ ) and a whole exome sequencing approach, two maternally inherited $\mathrm{X}$-linked variants in SHROOM4 and in ZFX were identified in a patient with an idiopathic neurodevelopmental phenotype that clinically overlap with Rett syndrome [6].

Recently, two maternally inherited interstitial microdeletions Xp11.22 including the CLCN5 and SHROOM4 genes were identified in a male patient [7]. This was the first report of this specific microdeletion in a patient presented with Dent disease, short stature and severe psychomotor delay. To address the question of the full spectrum of clinical variability, we analyzed a further patient with a maternally inherited microdeletion Xp11.22 including CLCN5 and SHROOM4.

\section{Case presentation}

The patient was a 4-year-old boy, the first child of non-consanguineous parents of Croatian origin. The family history was unremarkable. The pregnancy was complicated by polyhydramnios. The boy was delivered by caesarean section at 39 weeks of gestation with a length of $56 \mathrm{~cm}(+1.8 \mathrm{SD})$, weight of $3640 \mathrm{~g}(+0.4 \mathrm{SD})$ and occipitofrontal head circumference (OFC) of $35 \mathrm{~cm}$ (mean). The Apgar scores were 10, 10, and 10 at 1,5 , and $10 \mathrm{~min}$, respectively, and the umbilical arterial cord $\mathrm{pH}$ was normal with 7.30. After birth, physical examination showed a maldescensus of testes which was surgically corrected in the first year of life.

He started walking at 14 months. His speech development was delayed. At the age of 2 years and 6 months his speech and language development was assessed as that of a child of one year and 6 months. At 33 months, he was able to comprehend simple questions and commands and could speak two word sentences. At this time, his length was $84 \mathrm{~cm}(-3 \mathrm{SD})$, his weight was $11.7 \mathrm{~kg}(-2 \mathrm{SD})$ and his OFC was $45.5 \mathrm{~cm}(-3.8 \mathrm{SD})$. His facial features included thick and laterally broad eyebrows, wide set eyes, a short nose with a broad nasal bridge and nasal tip, epicanthus, a wide mouth with full lips, uplifted earlobes, low posterior hairline and dorsal hypertrichosis (Fig. 1). His fingers were short with bilateral clinodactyly $\mathrm{V}$. His toes were also short. At the age of 3 years, septic arthritis of the left hip occurred requiring antibiotic drug treatment. During this treatment a closure of the left femoral artery occurred which required surgical recanalization. X-ray examination of the hand showed that, according to Greulich and Pyle, his skeletal age of 1.5 years, at the chronological age of 2.5 years, was retarded. Furthermore,

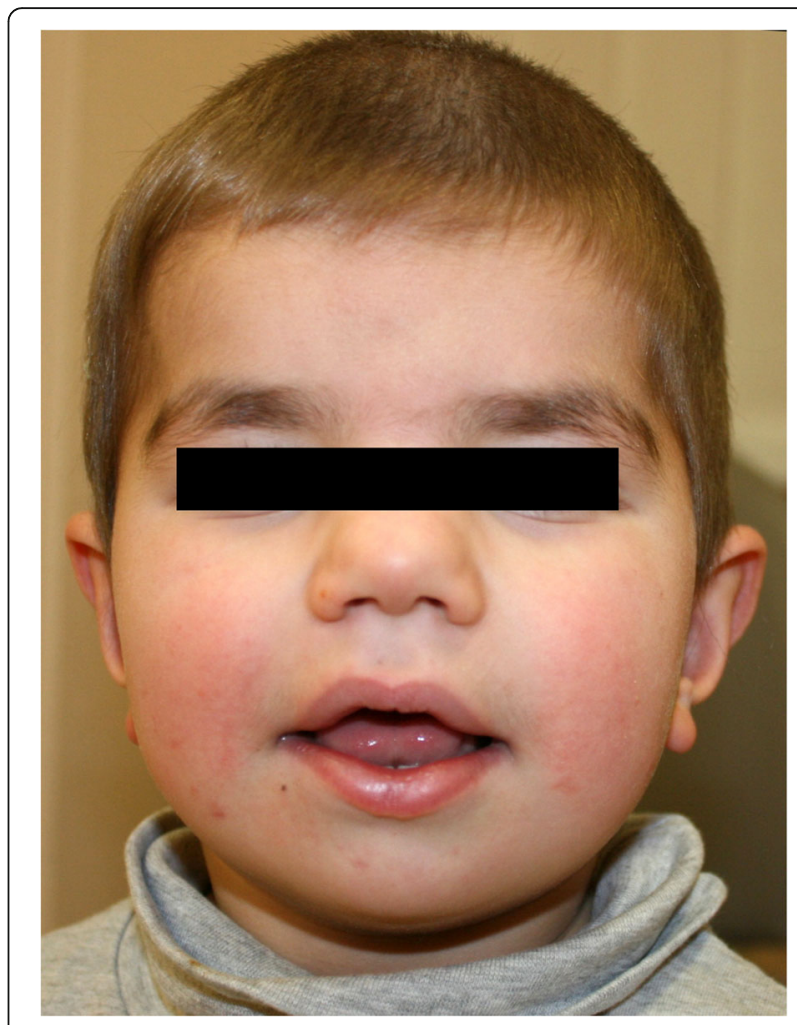

Fig. 1 Facial aspect of the patient with Xp11.22 microdeletion at the age of 4 years, showing laterally broad eyebrows, epicanthus, a short nose with a broad nasal bridge, a wide mouth with full lips, and uplifted earlobes

re-infected cancellous bone markings, cup-shaped metaphyses of the distal radius and ulna on the left with incipient sclerosis, only slight blurring and narrow cortex of the phalanges were detected, indicating compensated hypophosphatemic rickets.

At that time, abdominal ultrasound examination indicated that bilateral nephrocalcinosis was at stage IIa. Further diagnosis revealed partially generalized hyperaminoaciduria as seen in Fanconi syndrome, macroalbuminuria and hypophosphatemia (Additional file 1: Table S1). In addition, there was pronounced tubular proteinuria. The findings were compatible with tubulo-intestinal nephropathy. He was treated with an angiotensin-converting-enzyme inhibitor, he was supplemented with phosphate and vitamin D.

In the neuropediatric examination at the age of 4 4/12 years, the patient showed mild global developmental delay, including speech delay and mild intellectual disability. He was able to speak fluently in short sentences, but grammatical structure and articulation were reduced. Speech comprehension was good. Fine motor function was slightly reduced. He showed adequate social contact with good face-to-face-interaction and no sign of an autistic spectrum disorder. At this time, he received speech 
therapy and occupational therapy.. He achieved sphincter control during the day at the age of $33 / 12$ years, but had not achieved sphincter control during the night at the age of 4 4/12 years. Echocardiography showed no discernible valve defects. At the age of 4 4/12 years, his length was 92 $\mathrm{cm}(-3.2 \mathrm{SD})$, his weight was $14.0 \mathrm{~kg}(-1.81 \mathrm{SD})$, his OFC was $46.5 \mathrm{~cm}(-3.8 \mathrm{SD})$ and his body-mass-index was $16.5 \mathrm{~kg} / \mathrm{m} 2(+0.71 \mathrm{SD})$. His mother was tall $(180 \mathrm{~cm})$, had nystagmus but was otherwise healthy. Low-molecular proteinuria was found in her urine analysis.

The results of cytogenetic analysis of the index patient were normal. A microdeletion 22q11.2 was excluded by Fluorescence in situ Hybridization (FISH) analysis.

Standard cytogenetic analysis with a high resolution 550 GTG-banding was performed according to standard procedures, using a lithium-heparin peripheral blood sample from the patient and his mother.

High molecular weight genomic DNA from the patient was isolated from an EDTA peripheral blood sample (Qiagen, QIAamp DNA Blood Midi Kit) and processed according to manufacturer's protocol for the CytoScan $750 \mathrm{~K}$ assay. The genomic DNA was cut by restriction digest, adaptor-ligated and PCR-amplified. Purified amplicons were fragmented by DNAse digest, end-labeled with biotin and hybridized onto an array. After washing and staining, the array was scanned using the Affymetrix GCS3000Dx V2 system. The DNA sample from this patient was analyzed with the Affymetrix microarray platform (GeneChip System 3000Dx v.2). Primary Image Data were analyzed using the Chromosome Analysis Suite (ChAS) software version 3.1.1.27 with parameter settings allowing the detection of copy number changes of at least $50 \mathrm{~kb}$ and smaller and detection of contiguous regions of homozygosity $(\mathrm{ROH})>5 \mathrm{Mb}$. The copy number changes were analyzed by using multiple clinically relevant databases (e.g. DECIPHER, OMIM, DGV, UCSC, PubMed) and our own patient database. Interpretation and classification in terms of pathogenicity were carried out according to the American College of Medical Genetics guidelines [8] [9].

To clarify whether SHROOM4 is also affected by the microdeletion Xp11.23p11.22, we performed real-time quantitative PCR (qPCR) for the patient and his mother, measuring two amplicons covering the SHROOM4 and the CLCN5 genes. Genomic DNA from the patient and his mother was extracted from EDTA peripheral blood samples. LNA (locked nucleic acid) probes, contained within the Universal Probe Library, and primers were designed with the Roche Probe Finder Software. Real-time quantification of primer and probe pairs was carried out in comparison to a single-copy gene with efficiency correction using a calibrator normalization to determine the copy number. The qPCR analysis was performed on a Roche LightCycler v2 Instrument. The primer sequences are annotated in Additional file 1: Table S2.

Whole exome sequencing was performed on DNA extracted from blood. Library preparation of $100 \mathrm{ng}$ DNA was performed according to the manufacturer's protocol using SureSelect Human All Exon V6 (Agilent, Waldbronn Germany). Sequencing was performed on the Illumina HiSeq 2500 platform. Reads were aligned to the reference genome (Human Genome Assembly GRCh37) with Burrows-Wheeler Aligner (BWA) [10] and variants were called according to best practice guidelines [11]. For further analysis, all variants that have been reported in a homozygous state in more than three individuals in the cohorts of the $1 \mathrm{kGP}$ project, ExAC, gnomAD, or variants that have been classified as benign in ClinVar, were removed.

Variant analysis was carried out with GeneTalk [12]. Rare and coding, heterozygous, compound heterozygous, homozygous, and hemizygous variants in known ID (intellectual disability) genes were assessed with MutationTaster [13].

While the standard cytogenetic analysis showed a normal male karyotype in this patient, we identified, by molecular karyotyping, a hemizygous interstitial microdeletion Xp11.23p.11.22 of about $700 \mathrm{~kb}$, according to HG19 arr Xp11.23p.11.22 (49,649,226_50,351,579)×0 (Fig. 2a).

The genome coordinates chrX: 49649226_50,351,579 in the UCSC human genome 19 indicated that the deletion included CLCN5, the last three exons of SHROOM4 and four additional genes (Fig. $2 \mathrm{~b}$ and c).

For confirmation, we used target-specific qPCR analysis and validated this microdeletion, including a deletion of SHROOM4 in the patient and his mother. The coordinates for the minimum and maximum deletion intervals are chrX: $49649226-50,351,579 \mathrm{bp}$ and chrX:. 49648874-50,358,217 bp, respectively.

To rule out mutations in known genes related to intellectual disability, whole exome sequencing was performed. No known or predicted pathogenic mutations, that could explain the phenotypic features, were identified. However, the alignment of reads on chromosome $\mathrm{X}$ confirmed the deletion identified by array CGH.

\section{Discussion and conclusions}

This report presents a familial interstitial microdeletion of Xp11.23p.11.22 in a male patient with delayed speech development, mild intellectual disability, growth retardation, microcephaly, facial abnormalities and a renal phenotype with proteinuria, nephrocalcinosis and hypophosphatemia. Maternal transmission of this deletion was confirmed by qPCR tests. Among the six deleted genes, two are associated with diseases: The loss of CLCN5 could explain his renal phenotype, and SHROOM4 is a possible candidate gene for at least some of the other clinical manifestations. 


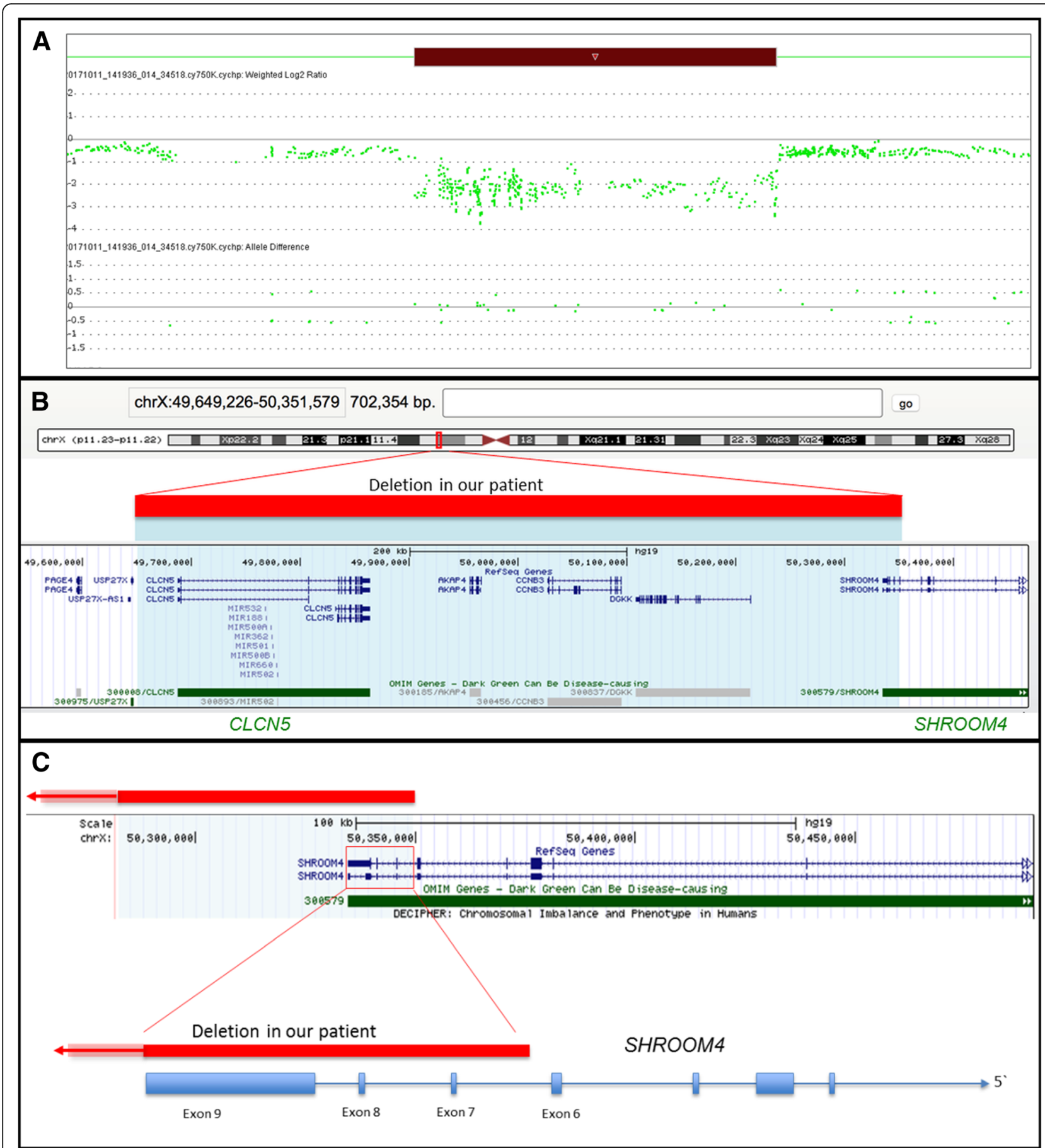

Fig. 2 a. Deletion in our patient indicated by red bar by ChAS Software. Decreased weighted Log2Ratios and collapsed allele difference tracks point to a hemizygous deletion (copy number 0) spanning genomic position 49,649,22,650,351,579 bp (hg19). b. Deletion in our patient (red bar) covering two disease-related genes: CLCN5 and parts of SHROOM4 (green bar) and three other genes (AKAP4, CCNB3, DGKK). c. Deletion in our patient (red bar) encompasses last coding exons of SHROOM4

SHROOM4 has been shown to be associated with $\mathrm{X}$-linked mental retardation [4]. Affected males from one family with a missense variant of this gene show severe intellectual disability, bilateral congenital hip luxation, and short stature [14]. Recently, a study of 19 patients with Rett-like phenotypes, identified two X-linked variants in SHROOM4 and ZFX in one individual [6]. This patient was a 14-year-old boy, and had severe intellectual disability with microcephaly, peripheral vasomotor disturbances, an attenuated response to pain, and autistic traits. 
Table 1 Comparison of two male patients affected with Xp11.22 microdeletion including SHROOM4 and CLCN5

\begin{tabular}{|c|c|c|}
\hline & $\begin{array}{l}\text { Patient reported by } \\
\text { Armanet et al. }\end{array}$ & $\begin{array}{l}\text { Patient reported } \\
\text { here }\end{array}$ \\
\hline Xp11.22 microdeletion & + & + \\
\hline Age & 4.5 years & 4 years \\
\hline Speech delay & Severe & + \\
\hline Global developmental delay & + & mild \\
\hline Other neurological abnormalities & Hydrocephalus & - \\
\hline Short stature & + & + \\
\hline Microcephaly & - & + \\
\hline Facial dysmorphisms & + & + \\
\hline Dent disease & + & + \\
\hline
\end{tabular}

In addition, dyspraxic gait, severe speech delay, eye pointing, and kyphosis were observed. To date, $Z F X$ has been shown to be involved in human sex determination and to be associated with retinoschisis and the phenotype of Turner syndrome, and thought to be a candidate gene for non syndromic primary ovarian insufficiency [15, 16]. A link between the $Z F X$ variant and the clinical manifestations of the reported patient is not known at present. Therefore this phenotype could be explained by the second X-linked variant in SHROOM4.

To date, only one male patient has been reported to carry two neighboring Xp11.22 microdeletions, including CLCN5 and SHROOM4 [7]. The microdeletions were detected by array CGH $(180 \mathrm{~K})$ and one had a size of $148 \mathrm{~kb}$ and included the CLCN5 gene and a second gene. The other microdeletion from the described patient had a size of $2.6 \mathrm{Mb}$ and contained 23 genes including SHROOM4. This $4 \frac{1}{2}$ year-old patient exhibited severe psychomotor delay, especially retarded speech development as well as short stature, facial dysmorphism, including a large forehead, and renal tubulopathy. Furthermore, he developed hydrocephalus due to stenosis of the aquaeduct of Sylvius.

Compared to the clinical findings in our patient, there is a clear overlap with regard to speech and developmental delay, short stature and the renal phenotype (Table 1). Mild craniofacial anomalies present in both include laterally broad eyebrows, and a short nose with a broad nasal bridge. The additional findings of hydrocephalus in the reported patient and microcephaly in our patient may be part of the clinical spectrum associated with this microdeletion.

There is a considerable clinical overlap with patients affected by the oculocerebrorenal syndrome, also referred to as Lowe syndrome. The oculocerebrorenal syndrome caused by OCRL1 mutations is mainly characterized by the triad congenital cataracts, intellectual disability and renal tubular dysfunction. However, the ocular manifestations can be missing or can occur later in life, in the second or third decade. Therefore, the oculocerebrorenal syndrome represents an important differential diagnosis of the condition described here [17].

The known Xp11.23p11.2 microduplication can be divided into the $4.5 \mathrm{Mb}$ recurrent duplication which includes the SHROOM4 gene, and atypical microduplications of different sizes. Within the recurrent $4.5 \mathrm{Mb}$ microduplication the SHROOM4 gene is considered to be one of the candidate genes for intellectual disability. In addition to intellectual disability carriers of this microduplication are affected by seizures and early onset of puberty in female patients [18]. In summary, our study shows that haploinsufficiency of CLCN5 and SHROOM4 is associated with tubulopathy and a syndromic form of ID with microcephaly and facial dysmorphisms, as well as short stature. These findings further expand the clinical spectrum of patients with Xp11.22 deletions.

\section{Additional file}

Additional file 1: Table S1. Quantitative findings on renal function. Abnormal laboratory parameters are bolded. All parameters were assessed during therapy with Ramiril 1.25 mg. Table S2. Primer Sequences and localisation of qPCR amplicons. (DOCX $16 \mathrm{~kb}$ )

\section{Abbreviations \\ Array CGH: Array-based Comparative Genomic Hybridization; Bp: Base pair; BWA: Burrows-Wheeler Aligner; FISH: Fluorescence in situ Hybridization; GRCh37, HG19: Human Genome Assembly GRCh37; ID: Intellectual disability; Kb: Kilo-base pair; LNA: Locked nucleic acid; OFC: Occipitofrontal circumference; qPCR: Real-time quantitative PCR; SD: Standard deviation}

\section{Acknowledgements \\ We would like to thank the family for their kind collaboration and contribution to this project.}

\section{Funding}

This work was not supported by grants.

Availability of data and materials

All data is available upon request.

Authors' contributions

MD contributed to manuscript preparation. EKS performed analyses. VR and JG did clinical evaluations. AK performed experiments and analyzed data. DH designed the study and wrote the manuscript. All authors read and approved the final manuscript.

\section{Ethics approval and consent to participate}

This study was approved by the Institutional Ethics Board of the Charite Universitätsmedizin Berlin. Written informed consent to participate was obtained from the parents on behalf of the patient.

\section{Consent for publication}

Written informed consent for the publication of this case report was obtained from the parents on behalf of the patient. A copy of the written consent is available for review by the journal editor. All authors have approved this manuscript.

Competing interests

The authors declare that they have no competing interests. 


\section{Publisher's Note}

Springer Nature remains neutral with regard to jurisdictional claims in published maps and institutional affiliations.

\section{Author details}

'Institute of Medical Genetics and Human Genetics, Charité -

Universitätsmedizin Berlin, corporate member of Freie Universität Berlin, Humboldt-Universität zu Berlin, and Berlin Institute of Health, Berlin, Germany. ${ }^{2}$ Praxis für Humangenetik-Friedrichstrasse, Berlin, Germany. ${ }^{3}$ Department of Pediatric Neurology, Sozialpädiatrisches Zentrum (SPZ), Center for Chronically Sick Children, Charité - Universitätsmedizin Berlin, Germany, corporate member of Freie Universität Berlin, Humboldt-Universität zu Berlin, and Berlin Institute of Health, Berlin, Germany. ${ }^{4}$ Department of Pediatric Nephrology, Charité - Universitätsmedizin Berlin, corporate member of Freie Universität Berlin, Humboldt-Universität zu Berlin, and Berlin Institute of Health, Berlin, Germany. ${ }^{5}$ Institute for Genomic Statistics and Bioinformatics, University Hospital Bonn, Berlin, Germany.

Received: 10 October 2018 Accepted: 28 December 2018

Published online: 10 January 2019

\section{References}

1. Dent CE, Friedman M. Hypercalcuric rickets associated with renal tubular damage. Arch Dis Child. 1964;39:240-9.

2. Lloyd SE, Pearce SH, Fisher SE, Steinmeyer K, Schwappach B, Scheinman SJ, et al. A common molecular basis for three inherited kidney stone diseases. Nature. 1996;379(6564):445-9.

3. Hoopes RR Jr, Shrimpton AE, Knohl SJ, Hueber P, Hoppe B, Matyus J, et al. Dent disease with mutations in OCRL1. Am J Hum Genet. 2005;76(2): 260-7.

4. Hagens O, Dubos A, Abidi F, Barbi G, Van Zutven L, Hoeltzenbein M, et al. Disruptions of the novel KIAA1202 gene are associated with X-linked mental retardation. Hum Genet. 2006;118(5):578-90.

5. Yoder M, Hildebrand JD. Shroom4 (Kiaa1202) is an actin-associated protein implicated in cytoskeletal organization. Cell Motil Cytoskeleton. 2007;64(1):49-63.

6. Lopes F, Barbosa M, Ameur A, Soares G, de Sa J, Dias Al, et al. Identification of novel genetic causes of Rett syndrome-like phenotypes. J Med Genet. 2016:53(3):190-9.

7. Armanet N, Metay C, Brisset S, Deschenes G, Pineau D, Petit FM, et al. Double Xp11.22 deletion including SHROOM4 and CLCN5 associated with severe psychomotor retardation and Dent disease. Mol Cytogenet. 2015;8:8.

8. Kearney HM, Thorland EC, Brown KK, Quintero-Rivera F, South ST. Working Group of the American College of medical genetics laboratory quality assurance C. American College of Medical Genetics standards and guidelines for interpretation and reporting of postnatal constitutional copy number variants. Genet Med. 2011;13(7):680-5.

9. de Leeuw N, Dijkhuizen T, Hehir-Kwa JY, Carter NP, Feuk L, Firth HV, et al. Diagnostic interpretation of array data using public databases and internet sources. Hum Mutat. 2012;33(6):930-40.

10. Li H, Durbin R. Fast and accurate short read alignment with burrowswheeler transform. Bioinformatics. 2009;25(14):1754-60.

11. McKenna A, Hanna M, Banks E, Sivachenko A, Cibulskis K, Kernytsky A, et al. The genome analysis toolkit: a MapReduce framework for analyzing next-generation DNA sequencing data. Genome Res. 2010; 20(9):1297-303.

12. Kamphans T, Krawitz PM. GeneTalk: an expert exchange platform for assessing rare sequence variants in personal genomes. Bioinformatics. 2012; 28(19):2515-6.

13. Schwarz JM, Cooper DN, Schuelke M, Seelow D. MutationTaster2: mutation prediction for the deep-sequencing age. Nat Methods. 2014; 11(4):361-2

14. Stocco dos Santos RC, Castro NH, Lillia Holmes A, Becak W, Tackels-Horne D, Lindsey CJ, et al. Stocco dos Santos X-linked mental retardation syndrome: clinical elucidation and localization to Xp11.3-Xq21.3. Am J Med Genet A. 2003;118A(3):255-9.

15. Simpson JL. Genetic and phenotypic heterogeneity in ovarian failure: overview of selected candidate genes. Ann N Y Acad Sci. 2008;1135:146-54.

16. Fortuno C, Labarta E. Genetics of primary ovarian insufficiency: a review. J Assist Reprod Genet. 2014;31(12):1573-85.
17. Bokenkamp A, Ludwig M. The oculocerebrorenal syndrome of Lowe: an update. Pediatr Nephrol. 2016;31(12):2201-12.

18. Nizon M, Andrieux J, Rooryck C, de Blois MC, Bourel-Ponchel E, Bourgois B, et al. Phenotype-genotype correlations in 17 new patients with an Xp11. 23p11.22 microduplication and review of the literature. Am J Med Genet A. 2015;167A(1):111-22

\section{Ready to submit your research? Choose BMC and benefit from:}

- fast, convenient online submission

- thorough peer review by experienced researchers in your field

- rapid publication on acceptance

- support for research data, including large and complex data types

- gold Open Access which fosters wider collaboration and increased citations

- maximum visibility for your research: over $100 \mathrm{M}$ website views per year

At $\mathrm{BMC}$, research is always in progress.

Learn more biomedcentral.com/submissions 